\title{
A!
}

This is an electronic reprint of the original article.

This reprint may differ from the original in pagination and typographic detail.

Nieminen, Kaarlo; Testova, Lidia; Paananen, Markus; Sixta, Herbert

\section{Novel insight in carbohydrate degradation during alkaline treatment}

Published in:

Holzforschung

DOI:

10.1515/hf-2014-0306

Published: 01/01/2015

Document Version

Publisher's PDF, also known as Version of record

Please cite the original version:

Nieminen, K., Testova, L., Paananen, M., \& Sixta, H. (2015). Novel insight in carbohydrate degradation during alkaline treatment. Holzforschung, 69(6), 667-675. https://doi.org/10.1515/hf-2014-0306

This material is protected by copyright and other intellectual property rights, and duplication or sale of all or part of any of the repository collections is not permitted, except that material may be duplicated by you for your research use or educational purposes in electronic or print form. You must obtain permission for any other use. Electronic or print copies may not be offered, whether for sale or otherwise to anyone who is not an authorised user. 
Kaarlo Nieminen*, Lidia Testova, Markus Paananen and Herbert Sixta

\section{Novel insight in carbohydrate degradation during alkaline treatment}

\begin{abstract}
A mathematical model is presented, in which the yield loss (YL) and the decline in polymerization of carbohydrates is comprehended. The model is applicable to the treatment of cellulose and hemicelluloses in alkaline media, and it features the actions of peeling, stopping, and alkaline hydrolysis of the polymer chains. The peeling reaction is further subdivided into primary and secondary peeling depending on whether it originates from an initial reducing end-group (REG) or from an REG created by alkaline hydrolysis. Fitting the model to experimental data provides estimates of the various reaction rate constants. When available, simultaneous observations of the YL and the decrease in chain length contribute to the evaluation of the parameters. Alternatively, if the data are limited to the YL, the obtained parameter estimates allow for a projection of the time development of chain length. The model has been applied on data from two types of experiments: soda-anthraquinone treatment of cotton linters and kraft treatment of Scots pine. It was possible to evaluate the impact of the different processes on degradation as well as the portions of polymer chains possessing active or stabilized REGs.
\end{abstract}

Keywords: alkaline hydrolysis, alkaline treatment, biorefineries, carbohydrates, cotton linter, degradation, degree of polymerization, mathematical model, peeling, reaction kinetics, reducing end-group, Scots pine, stopping, yield loss

DOI 10.1515/hf-2014-0306

Received October 15, 2014; accepted February 4, 2015; previously published online $\mathrm{xx}$

\section{Introduction}

Peeling, stopping, and alkaline hydrolysis are central reactions involved in the degradation of carbohydrate

*Corresponding author: Kaarlo Nieminen, Aalto University, P.O. Box 16400, 00076 Aalto, Finland, e-mail: Kaarlo.Nieminen@aalto.fi Lidia Testova, Markus Paananen and Herbert Sixta: Aalto University, P.O. Box 16400, 00076 Aalto, Finland chains, such as cellulose, (galacto)glucomannan (GGM), and xylan (Haas et al. 1967; Lai and Sarkanen 1967). There are several studies on the interplay between those reactions in the context of disposal of nuclear waste, where the focus has been on the degradation of cellulosic material under moderate ambient conditions over the course of thousands or millions of years (Van Loon and Glaus 1997; Glaus et al. 1999; Knill and Kennedy 2003; Pavasars et al. 2003; Glaus and Van Loon 2008; Rout et al. 2014). However, it seems that modeling of the same reactions under conditions relevant to biorefineries has received far less attention.

Sjöström (1977) untangled the reaction pathways of the polysaccharides degrading into low molecular weight products during alkaline pulping. De Groot et al. (1995) worked with hemp woody core and applied a first-order model divided into stages of different reactivities on carbohydrate degradation. Van Heiningen et al. $(2002,2004)$ employed cellulose mass balance during degradation to derive a relationship between alkaline pulp yield and the degree of polymerization (DP) of cellulose in pulp. Shatalov and Pereira (2005a,b) investigated polysaccharide degradation during ethanol-alkali delignification and developed a kinetic model, where each polysaccharide component is divided into a fast and a slow degrading fraction. Paananen et al. (2010) studied the behavior of wood components during kraft pulping in the range of $80^{\circ} \mathrm{C}-130^{\circ} \mathrm{C}$ and presented kinetic models, based on the end-wise reactions, for the degradation of the components. Pakkanen et al. (2013) investigated the dependence of the carbohydrate degradation products on the cooking parameters during kraft pulping. Studies by Longue et al. (2013) and Wei et al. (2013) considered the extraction of hemicelluloses from eucalyptus wood through alkaline treatment. Van Heiningen and Ji (2012) and Jafari et al. (2014) inspected the combined effect of kraft pulping followed by oxygen delignification on the carbohydrate yield. In the present study, a mathematical model incorporating peeling, stopping, and alkaline hydrolysis is employed to describe the degradation of carbohydrates in biorefineries during alkaline treatment. A model is sought for, which is able to consider two different aspects of the degradation: the yield loss (YL) and the decrease in polymerization (DecP). 
Cellulose and hemicelluloses consist of long chains of anhydro-monosaccharide units. Cellulose is a linear homopolymer made up of anhydro-glucose units, whereas hemicelluloses are slightly branched hetero-copolymers. Wood cellulose has average DPs in the range of 5000-10 000, whereas hemicelluloses have DPs approximately 100-200 (Sjöström 1993; Sixta et al. 2006). One chain end of cellulose and hemicelluloses is reducing and the other is nonreducing. Further, the reducing endgroup (REG) can be either active or stabilized. If a chain has an active REG, the end-monosaccharide unit can be eliminated from the chain by the so-called peeling reaction. The subsequent element in the chain then forms a new active REG admitting further peeling. Within such a sequence of REGs, where the elimination of one REG leads to the creation of the next, the previous REGs are called ancestors in relation to the later ones (descendants). The peeling reaction plays a central role in the degradation of the polysaccharide chains and the resulting loss of yield during aqueous alkaline conditions. The stopping reaction terminates the end-wise peeling by stabilizing the REG. These two reactions alone would, after some time, lead to a situation where all the active REGs are stabilized and degradation is terminated. However, the reaction of alkaline hydrolysis alters the scene by eliminating a non-end element, thus cleaving the chain. One of the new chain endunits then becomes an active REG again susceptible to the peeling reaction, whereas the other becomes a non-REG. Peeling descending from initially active REGs is classified as primary peeling, whereas peeling as a result of alkaline hydrolysis is classified as secondary peeling. Testova et al. (2014) and Mozdyniewicz et al. (2013) presented a mathematical model for the carbohydrate YL, considering the interplay between the relevant degradation reactions. The first quoted paper focused on the effects of acid pretreatment and subsequent stabilization on cotton linters pulp, in which the fitting of the degradation model to the various pretreatment and stabilization reactions resulted in different reaction rate estimates for each case.

The application of the YL model to kraft-treated Scots pine data provided an evaluation of the impacts of the various reactions on the YL, showing that primary peeling accounts for most of the rapid initial YL during alkaline treatment (Nieminen et al. 2014). After the stabilization of the primary REGs, secondary peeling dominates the YL. The direct contribution of alkaline hydrolysis to YL is very small, but that caused by secondary peeling is significant.

The DecP is often considered independently of the YL, with emphasis on the role of alkaline hydrolysis. Ekenstam (1936) developed a model for the DecP in terms of the rate constant of hydrolysis. Calvini (2012) gives an alert account for the further development of the still relevant Ekenstam equation.

In the present study, the carbohydrate degradation model should be augmented to incorporate also the DP. It is well understood that the end-wise reactions on the carbohydrates during alkaline treatment affect not only the yield but also more specifically the DP of the carbohydrates. The peeling reaction shortens the chain by removing elements, one at time, from the reducing end, and alkaline hydrolysis, more drastically, by splitting the chain in two shorter parts. To quantify the DP development theoretically, data from the studies of Testova et al. (2014) and Paananen et al. (2013) will be applied and a mathematical model will be developed for the DecP during alkaline treatment comprising the same parameters as the YL model. In the case that experimental data are available for the DecP data, the model can enhance the estimates of the YL model. On the contrary, if only YL data are available, fitting the YL model data into the DP model will still provide a means for simulating the DP development. The objective is also to compare the contributions of the different reactions to the DecP.

\section{Materials and methods}

Two categories of data were utilized in this study. First, sodaanthraquinone (AQ) treatment of cotton linters (Testova et al. 2014), which permits the easy evaluation of the DP because of the absence of lignin. Second, the raw material from Scots pine was subjected to kraft treatment (Paananen et al. 2013), producing degradation data on glucomannan, xylan, and cellulose.

Soda-AQ treatment of cotton linters: Cotton linter pulp was ground in a Wiley mill with a mesh size of $0.5 \mathrm{~mm}$. In this context, four types of experiments were carried out, with regard to acid pretreatment and stabilization: (1) no pretreatment (CL), (2) pretreatment with oxalic acid (OA), (3) pretreatment with oxalic combined with borohydride (BH) stabilization attempted in situ in the alkaline degradation stage $(\mathrm{OA}+\mathrm{BH})$, and (4) pretreatment with $\mathrm{OA}$ combined with $\mathrm{AQ}$-2-sulfonic acid sodium (AQS) salt stabilization attempted in situ in the alkaline degradation stage (AQS). The acid pretreatment took place at a $15 \mathrm{ml} \mathrm{g}^{-1}$ liquid-to-solid ratio at $110^{\circ} \mathrm{C}$. The isothermal duration was $80 \mathrm{~min}$. The alkaline degradation trials were conducted at a temperature of $160^{\circ} \mathrm{C}$, a liquid-to-solid ratio of $40 \mathrm{ml} \mathrm{g}^{-1}$, an alkali concentration of $20 \mathrm{~g} \mathrm{l}^{-1}$, and the addition of dispersed $0.1 \mathrm{~g} \mathrm{l}^{-1} \mathrm{AQ}$. The content of REGs was evaluated using the bicinchoninic acid method. The cotton linters experiments are described in detail by Testova et al. (2014).

Kraft treatment: The wood material was chipped and screened according to SCAN-CM 40:01. Subsequently, the screened chips were milled with a Wiley mill to pass through a $1 \mathrm{~mm}$ slot screen. The experiments were conducted in a 101 batch reactor at a liquorto-wood-ratio of 200:1. The $\mathrm{OH}$ ion concentration levels were 0.5 and $1.55 \mathrm{M}$. For the lower $[\mathrm{OH}]$ level, the temperature was $160^{\circ} \mathrm{C}$, and at 
the higher $[\mathrm{OH}]$ level, cooks were performed at $130^{\circ} \mathrm{C}, 140^{\circ} \mathrm{C}, 150^{\circ} \mathrm{C}$, or $160^{\circ} \mathrm{C}$. The addition of $\mathrm{NaCl}$ fixed the ionic strength at a constant level of $2.00 \mathrm{M}\left[\mathrm{Na}^{+}\right]$. For all cooks, the sulfidity was kept at $33 \%$. At each $\left[\mathrm{OH}^{-}\right]$level, a temperature combination of a series of approximately 10 cooks with increasing duration was performed, the shortest lasting under $10 \mathrm{~min}$ and the longest lasting for a few hours. Both the wood residue and the black liquor were analyzed for carbohydrate content. The Scots pine experiments are described in detail by Paananen et al. (2013).

Mathematical models: Model for YL: The system of differential equations below describes the joint effects of the different end reactions on a carbohydrate molecule (Mozdyniewicz et al. 2013; Nieminen et al. 2014; Testova et al. 2014):

$$
\left\{\begin{array}{l}
\frac{d R}{d t}=-k_{s} R+k_{h}\left(\Gamma_{0}-P-H\right) \\
\frac{d P}{d t}=k_{P} R \\
\frac{d H}{d t}=k_{h}\left(\Gamma_{0}-P-H\right)
\end{array}\right.
$$

Here, $R$ is the amount of active REGs, $P$ is the material degraded by peeling, and $H$ is the material degraded by alkaline hydrolysis. The amount of initial material is denoted by $\Gamma_{0}$ and the reaction rate constants for peeling, stopping, and alkaline hydrolysis are designated as $k_{\mathrm{p}}, k_{\mathrm{s}}$, and $k_{\mathrm{h}}$, respectively. The first term on the right in the first equation of the Equation (1) system just indicates the decrease rate in active REGs due to the stopping reaction, and the second equation describes that the peeling rate is proportional to the amount of active REGs. That the second term on the right of the first equation in the system is the same as that on the right of the third equation reflects the fact that each alkaline hydrolysis reaction creates a new active REG. It is possible to transform the system of differential equations to a second-order differential equation, from the analytic solution, of which expressions for $R, P$, and $H$ can be obtained. Alternatively, one can work with a numerical solution of the system. The sum $P+H$ then gives the model function to be compared with the experimentally obtained YL.

The parameters related to the Equation (1) system are (a) the initial values for the amounts of peeled-off material $(P)$ and the material removed by alkaline hydrolysis $(H)$ as well as the initial portion of REGs and (b) the reaction rate constants for peeling, stopping, and alkaline hydrolysis.

Assuming that no peeling or alkaline hydrolysis has occurred before the alkaline treatment, the initial values of $P$ and $H$ are zero. It is, however, less obvious what the initial amount of the active REGs is. Although some estimates of the average chain lengths for cellulose and the hemicelluloses exist, the knowledge about the division of the REGs into active and stabilized ones is deficient. Hence, the reciprocal of the chain length only gives an upper limit to the number of active REGs. Therefore, the initial number of active REGs and the reaction rate constants are left as free parameters to be determined through a fit of the model to experimental degradation data.

Fitting the mathematical model to the experimental data gives estimates for the reaction rates. The presence of several different temperatures in the experimental data enables the calculation of the frequency factors and activation energies for the reactions according to the well-known Arrhenius equation. The equation system can be modified to describe separately the portions of primary and secondary peeled carbohydrate materials (Nieminen et al. 2014).
Model for DecP: A peeling reaction reduces the polymer chain length by one element, whereas an alkaline hydrolysis reaction, apart from removing one element from the chain, also splits the chain in two parts, thus, on the average, reducing the chain to half of its previous length. Correspondingly, $n$ cases of alkaline hydrolysis reaction on a polymer chain divide the original chain in $n+1$ parts, thus decreasing the length by the same factor. Hence, the DP can be expressed as a function of the original chain length and the numbers of the different peeling and alkaline hydrolysis reactions:

$$
D P=\frac{n_{0}-n_{P}-n_{H}}{1+n_{H}}
$$

Here, $n_{0}$ is the length of the initial chain, $n_{p}$ is the number of peeling reactions, and $n_{\mathrm{H}}$ is the number of alkaline hydrolysis reactions.

To express the DecP model with the same variables as the YL model, the quantities in Equation (1) can be related with those of Equation (2):

$$
\frac{n_{0}}{\Gamma_{0}}=\frac{n_{p}}{P}=\frac{n_{H}}{H}
$$

or

$$
n_{p}=\frac{n_{0}}{\Gamma_{0}} P
$$

and

$$
n_{H}=\frac{n_{0}}{\Gamma_{0}} H
$$

Inserting Equations (4) and (5) into Equation (2) and multiplying both the numerator and the denominator on the right with $\Gamma_{0} / n_{0}$ result in:

$$
D P=\frac{\Gamma_{0}-P-H}{\frac{\Gamma_{0}}{n_{0}}+H}
$$

In this version of the DecP model, the free parameters related to the reaction rates are the same as those in the YL model. In addition, the DecP model contains the parameter for the chain length $n_{0}$, the reciprocal of which gives an estimate for the total (both active and stabilized) number of REGs. In Equation (6), the amount of active REGs $R$ does not appear explicitly, but the initial value of $R$ is still present implicitly as $P, R$, and $H$ are all parts of the solution of the same system of differential equations [Equation (1)].

Combination of the YL and DecP models: Because the YL and DecP models both have the same sets of free parameters, fitting the models in two separate fits will give two different estimates for each parameter. Therefore, it would be adequate, in cases when experimental data for both models exist, to combine the two data sets and the two models would result in for a single estimate for each free parameter fitting both models simultaneously to their respective data. On the contrary, if only YL data are available, the free parameters can be estimated and subsequently the DecP can be simulated. For fitting, both YL and DecP data sets of cotton linters data were assembled into a common matrix with an added dummy column to indicate to which original data set a row belongs. In a similar way, four other dummy 
columns were introduced for the pretreatments to allow for fitting the model simultaneously to the data of the four different pretreatments, with rate constants independent of the pretreatments. Table 1 summarizes the dependence of the matrix element values on whether the data row represents YL or DecP values and also indicates the kind of pretreatment. In the last column with the response values, the rows associated to the DecP data have reciprocal DP values in accordance to that what is customary when fitting DP models (Calvini 2013). Further, the reciprocal DP value is multiplied with a weight coefficient $\mu$, to make the values commensurable with the YL values, and in this approach imposes equal significance on the two data sets with regard to the fit. The combined model function (Equation 7) was composed as a sum of the individual functions, where the coefficients of the terms depended on the dummy variables in such a way that the coefficient is 1 , when the corresponding data row is relevant for the term and zero otherwise.

$$
z=\sum_{i=1}^{4} \alpha_{i}\left((1-\lambda) \operatorname{YL}\left(\xi_{i}, k_{p}, k_{s}, k_{h}, t\right)+\frac{\mu \cdot \lambda}{D P\left(\xi_{i}, \eta_{i}, k_{p}, k_{s}, k_{h}, t\right)}\right)
$$

As before, the $k: s$ are the rate constants for the endwise reactions. Further, $\xi_{\mathrm{i}}$ and $\eta_{\mathrm{i}}$ denote the initial amounts of active and stabilized REGs, respectively. The subindex $i$ in these two quantities indicates that they change with the type of pretreatment as opposed to the rate constants. In agreement with the DecP-related values in the data matrix, the DecP term in the function appears as a reciprocal multiplied with the weight coefficient $\mu$. The combined model function has one nonzero term for each row because every row of the combined data matrix originates in either the YL or the DecP data and represents one and only one of the four pretreatment options. The Mathematica software function "NonlinearModelFit" provided parameter estimates and SEs by fitting the model function to the data.

Relationships between active REGs, stabilized REGs and DecP: In our model, a REG is either active or stabilized. Hence, the total number of REGs within an ensemble of macromolecules of a certain polysaccharide is the sum of the active and stabilized REGs:

$$
N(R E G)=N(\text { aREG })+N(s R E G)
$$

Here, aREG and sREG denotes active and stabilized REGs, respectively, and the $N$ prefix specifies that it is the plain number of the REGs within the ensemble that is under scrutiny. If the terms in

Table 1: Elements in the different columns of the combined data matrix.

\begin{tabular}{lll}
\hline & Elements & Values in different cases \\
\hline Dummy variables & $\lambda$ & 0 if YL data, 1 if DecP data \\
& $\alpha_{1}$ & 1 if $\mathrm{CL}^{\mathrm{a}}$ data, otherwise 0 \\
& $\alpha_{2}$ & 1 if $\mathrm{OA}^{\mathrm{b}}$ data, otherwise 0 \\
& $\alpha_{3}$ & 1 if $\mathrm{BH}^{\mathrm{c}}$ data, otherwise 0 \\
& $\alpha_{4}$ & 1 if $\mathrm{AQS}$ data, otherwise 0 \\
Response & $t$ & Treatment time value \\
& $z$ & YL data or DecP data \\
\hline
\end{tabular}

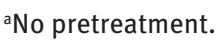

bPretreatment with $\mathrm{OA}$.

cPretreatment with $\mathrm{OA}$ and $\mathrm{BH}$ stabilization.

dPretreatment with $O A$ and $A Q S$.
Equation (8) are divided by the total number of fundamental repeating structure units of the polymer in the ensemble, the results is

$$
n(R E G)=n(a R E G)+n(s R E G)
$$

Here, the prefix $n$ stands for the number of REGs per fundamental repeating structure unit. Because every macromolecule has one and only one REG, $n$ (REG) is the reciprocal of the (number average of) the DP. Now, the initial DP was one of the parameters of the DecP model; in Equation (6), the initial DP was denoted by $n_{0}$. Further, the initial amount of active REGs was one the parameters in the YL model. Thus, if both YL and DecP development data are available, the initial $n$ (REG) and $n$ (aREG) can be estimated by fitting the combined YL and DecP model to the data, and from this, the initial n(sREG) can be simply calculated according to Equation (9). If the YL development data are available together with an a priori estimate for the initial average DP but no DP development data, the initial $n$ (aREG) can be estimated from fitting the YL model. Then, the initial $n$ (REG) can be obtained as the inverse of the initial DP; consequently, the initial $n$ (sREG) can be calculated from Equation (9).

If the initial $n$ (REG), $n$ (aREG), and $n$ (sREG) and the corresponding rate constants are estimated, the respective time developments can also be calculated, bearing in mind that the function $R$ of Equation (1) describes the an REG development and Equation (6) describes the DecP development. The sREG development follows from Equation (9).

The REG amounts will be expressed in the following as molality values (i.e., $\mu \mathrm{mol} \mathrm{g} \mathrm{g}^{-1}$ of the polymer):

$$
m(R E G)=10^{6} \times \frac{n(R E G)}{M}
$$

Here, $m(\mathrm{REG})$ is the molality of the REGs and $M$ is the molar mass $\left(\mathrm{g} \mathrm{mol}^{-1}\right)$ of the fundamental repeating structure unit of the polymer (162 $\mathrm{g} \mathrm{mol}^{-1}$ for cellulose as well as GGM and $132 \mathrm{~g} \mathrm{~mol}^{-1}$ for xylan). The factor $10^{6}$ is due to expressing the molalities in terms of micromole instead of mole.

\section{Results and discussion}

\section{Alkaline treatment of cotton linters pulp}

The experiments with cotton linters pulp provided both YL and DecP for the cellulose as the only fiber component. The fitting of the comprehensive model to these data confirmed the existence of common model parameter estimates for the YL and DecP models. Further, the same reaction rate constants for peeling, stopping, and alkaline hydrolysis could describe the YL and DecP developments, notwithstanding the type of pretreatment. Figure 1a and c shows the fits of the comprehensive model to the YL and DecP data, respectively. The partition of the degraded material into contributions of primary and secondary peeling as well as alkaline hydrolysis (Figure 1b) reveals that primary very fast peeling is restricted almost entirely to the first few minutes. Furthermore, secondary peeling dominates the 

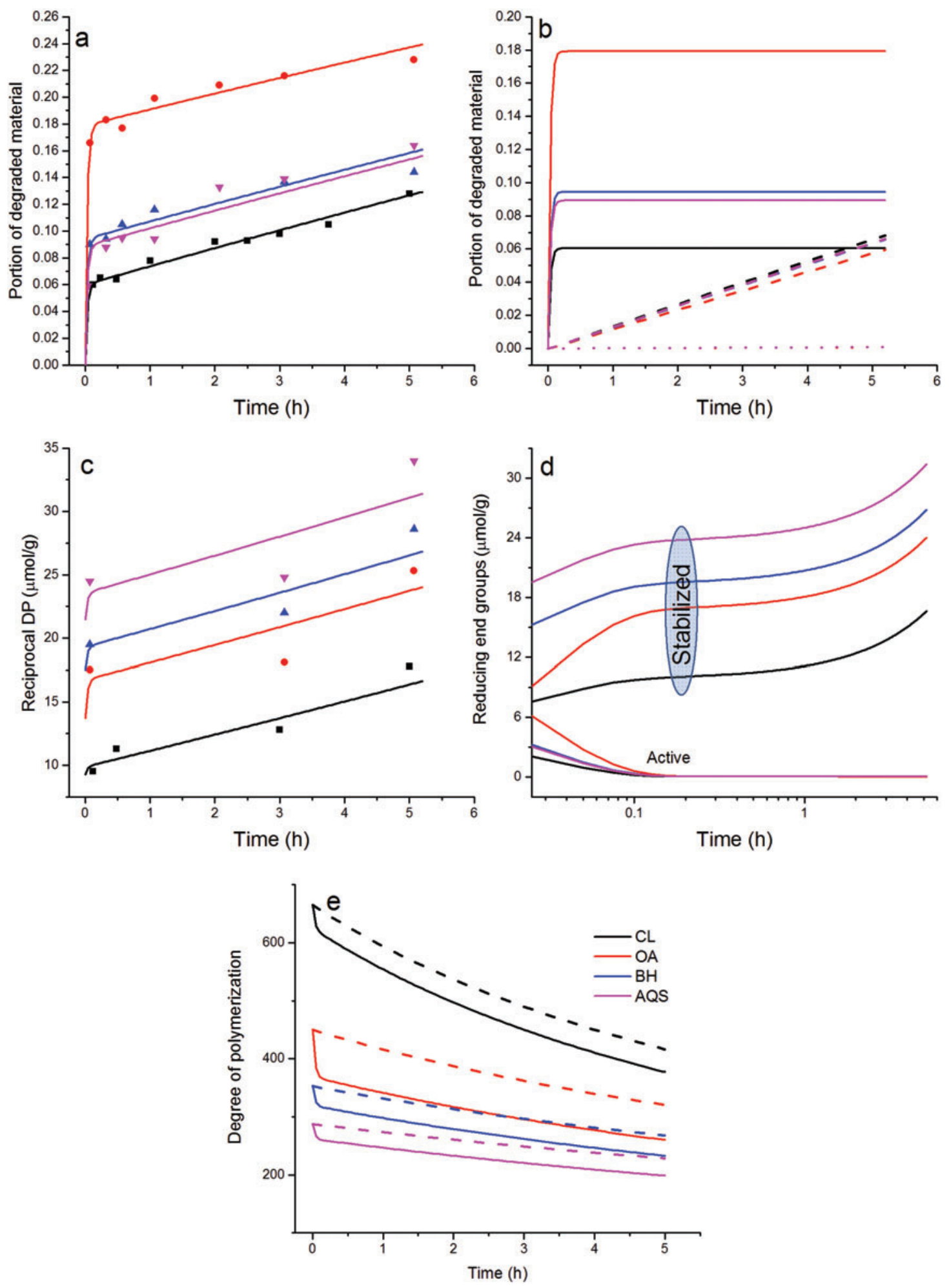

Figure 1: Cotton linter pulp degradation: (a) model fitted to YL data, (b) calculated cumulative portions of YL through primary peeling (solid lines), secondary peeling (dashed lines), and alkaline hydrolysis (dotted lines close to zero), (c) model fitted to DP data, (d) calculated development of the portions of active and stabilized REGs (logarithmic time scale); and (e) significance of peeling on DP in alkaline treatment of cotton linters pulp (solid lines: the DP model with the reaction rate constants obtained from the combined YL-DecP fit; dashed lines: the DP model where the peeling rate constant is set to 0 ). 
Table 2: Reaction rate constants for peeling $k_{\mathrm{p}}$, stopping $k_{\mathrm{s}}$, and alkaline hydrolysis $k_{\mathrm{h}}$ together with SEs estimated by nonlinear regression of the combined YL and DP models to the cotton linters kier boiling data. Temperature $160^{\circ} \mathrm{C}$, alkali concentration $0.5 \mathrm{M}$, liquid-to-solid ratio $40 \mathrm{ml} \mathrm{g}^{-1}$. Collective estimates for the four different types of pretreatment.

\begin{tabular}{lr}
\hline Rate constant & Estimated value \\
\hline$k_{\mathrm{p}}\left(\mathrm{h}^{-1}\right)$ & $2597 \pm 865$ \\
$k_{\mathrm{s}}\left(\mathrm{h}^{-1}\right)$ & $32.0 \pm 7.9$ \\
$k_{\mathrm{h}} \times 10^{3}\left(\mathrm{~h}^{-1}\right)$ & $0.179 \pm 0.025$ \\
$k_{\mathrm{p}} / k_{\mathrm{s}}$ & 81 \\
$k_{\mathrm{h}} / k_{\mathrm{s}} \times 10^{6}$ & 14.5 \\
\hline
\end{tabular}

Table 3: Initial REG molalities and SEs estimated by nonlinear regression of the combined YL and DP models to the cotton linters data. Temperature $160^{\circ} \mathrm{C}$, alkali concentration $0.5 \mathrm{M}$, liquid-to-solid ratio $40 \mathrm{ml} \mathrm{g}^{-1}$. Different estimates for different types of pretreatment. For the various pretreatments, see footnotes in Table 1.

\begin{tabular}{lrr}
\hline Pretreatment & Active REGs $\left(\mu \mathrm{mol} \mathrm{g}^{-1}\right)$ & Stabilized REGs $\left(\mu \mathrm{mol} \mathrm{g}^{-1}\right)$ \\
\hline $\mathrm{CL}$ & $4.6 \pm 1.1$ & $4.7 \pm 1.2$ \\
$\mathrm{OA}$ & $13.6 \pm 2.6$ & $0.1 \pm 2.9$ \\
$\mathrm{BH}$ & $7.2 \pm 1.6$ & $10.3 \pm 2.0$ \\
$\mathrm{AQS}$ & $6.8 \pm 1.5$ & $14.7 \pm 2.2$ \\
\hline
\end{tabular}

subsequent phase featuring nearly constant degradation. As for alkaline hydrolysis, its explicit contribution to the YL is insignificant. Finally, the estimated initial molalities of active and stabilized REGs show a distinct effect of the pretreatment, where the pulp treated with OA without any subsequent stabilization has the highest occurrence of active REGs, and an additional treatment with $\mathrm{BH}$ or AQS increases the number of stabilized REGs. The AQS treatment stabilized more REGs than did the BH treatment. Figure 1d displays the decrease in the active REGs and increase in the stabilized REGs during alkaline treatment. The order of the REG molalities for the four different pretreatments remains the same as initially for both active and stabilized REGs throughout the treatment period. Tables 2 and 3 summarize the parameter estimates of the combined model.

The respective effects of peeling and alkaline hydrolysis on DP have been investigated by computer simulation (in silico) switching off the peeling reaction. Figure 1e compares the DP development in the case that the peeling rate constant is set to zero with the case when it has the value determined by the fit of the combined model. In both cases, the parameter values of the other parameters are those determined by the fit. There is a clear difference
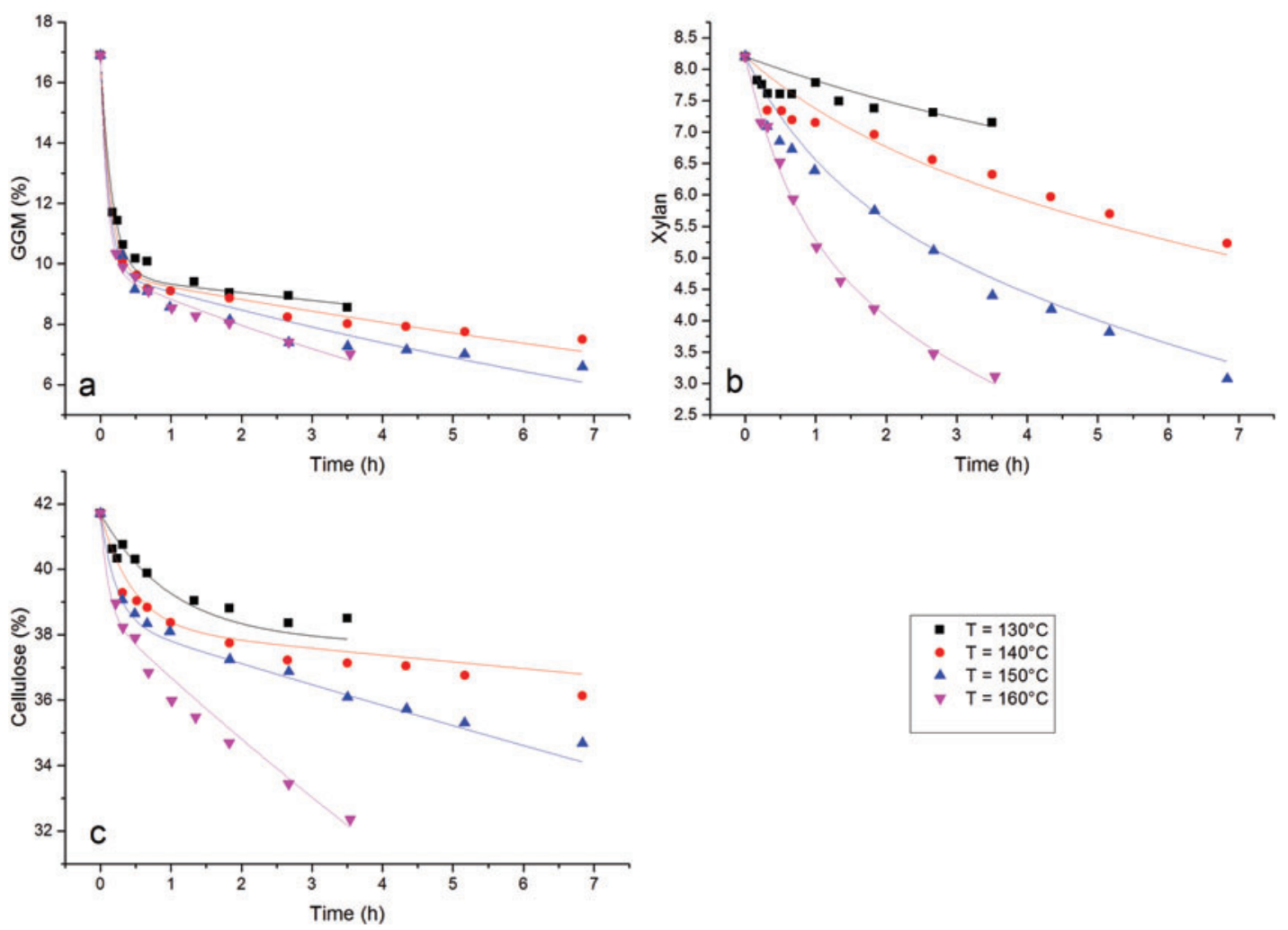

Figure 2: Fit of YL model to the amounts of (a) GGM, (b) xylan, and (c) cellulose remaining in either the wood residue or the black liquor during kraft cooking of Scots pine, at different temperatures.

Alkali concentration: $1.55 \mathrm{M}$ (figure adapted from Nieminen et al. 2014). 
between the two cases indicating, perhaps a bit conterintuitively, that the effect of peeling is indeed significant for the shortening of the polymer chains.

\section{Kraft treatment of Scots pine}

For the experiments with Scots pine, only the YL data were accessible. In a previous study (Nieminen et al. 2014), these data were employed to estimate the kinetic parameters associated to the degradation. Apart from the end-wise degrading reactions, which remove one element at a time from the polymer chains, the carbohydrates in the kraft cooking experiment can also be dissolved into the black liquor in longer fragments. To be able to fit the model expression for the remaining carbohydrates to the data, it is assumed that the end-wise reactions proceed with the same reaction rates in the black liquor as in the wood residue. The model was then fitted to the joint amount of carbohydrates in the wood residue and the black liquor. Figure 2 shows the fit of the YL model to the data for different carbohydrates and temperatures. Table 4 summarizes the estimated activation energies and frequency factors related to the various reactions. The several temperatures applied enable an estimation of the activation energies and frequency factors, from which the rate constants can be calculated through the Arrhenius equation. Further, estimates for the initial amount of active REGs were obtained through the fit. These estimates together with some a priori knowledge of the total initial amount of REGs (or equivalently the initial DP) will allow for the calculation of the amount of stabilized REGs. Sixta et al. (2006) reported the a viscosity average of 5080 was related to the DP. Assuming a polydispersity index of 3, this implicates a $\mathrm{DP}_{\mathrm{n}}$ of 1693 . The corresponding total initial fraction of REGs for cellulose is then 0.00059 , and this value was selected as an a priori estimate for the total REGs. As for GGM, the following estimates for the total initial fractions for GGM are available: 0.0071 (Procter and Apelt 1969), 0.0064 (Young and Liss 1978), and 0.0098
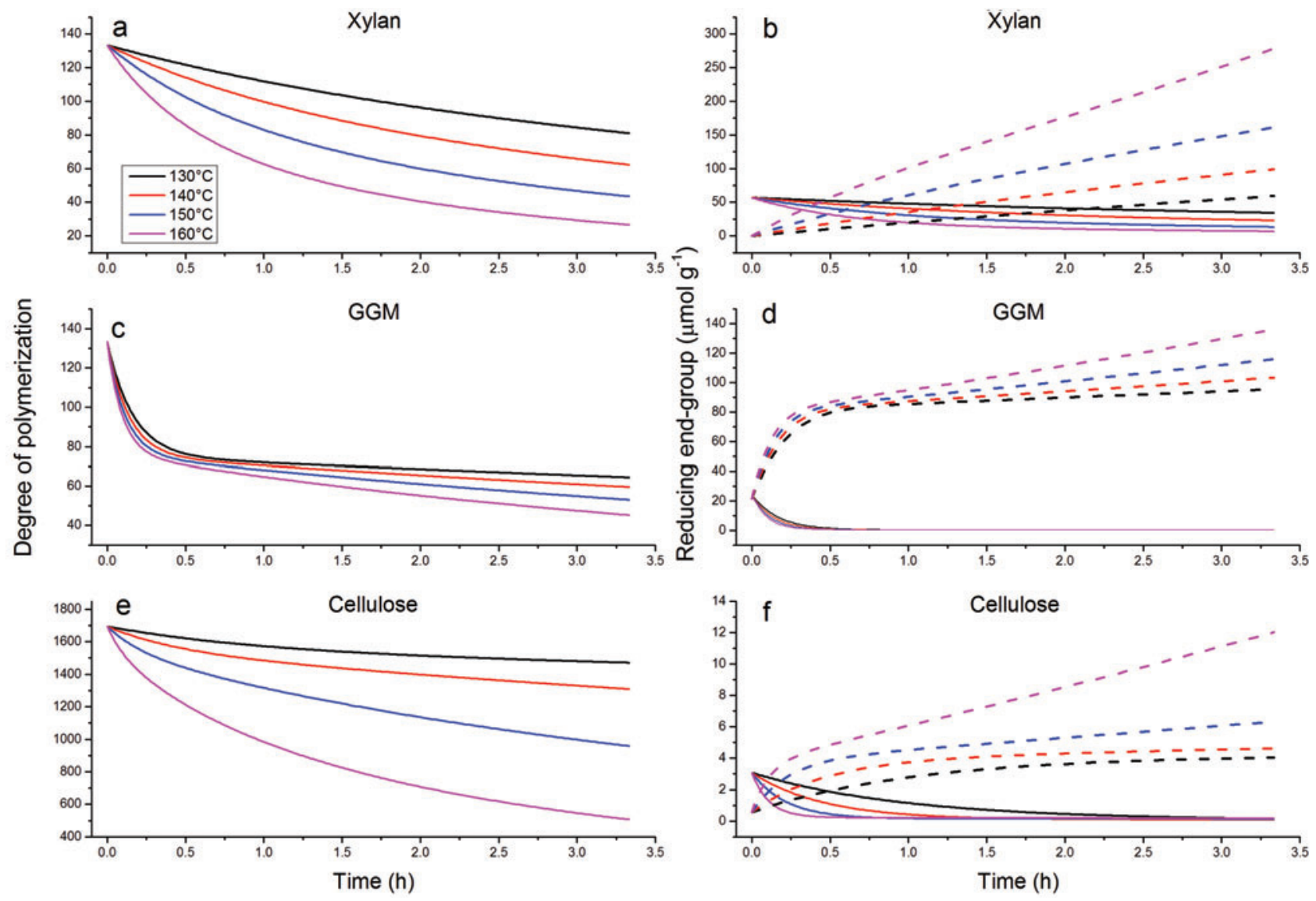

Figure 3: Simulated DP and REG for various carbohydrates during kraft treatment: (a) DP for xylan, (b) REG for xylan, (c) DP for GGM, (d) REG for GGM, (e) DP for cellulose, and (f) REG for cellulose.

In the REG subfigures (b), (d), and (f), solid lines denote active REGs and dashed lines denote stabilized REGs. 
Table 4: Estimated kinetic parameters in the Arrhenius equation related to the endwise reactions during kraft cooking of Scots pine at alkali concentration $1.55 \mathrm{M}$.

\begin{tabular}{lrrrr}
\hline Reaction & Parameter & GGM & Xylan & Cellulose \\
\hline Peeling & $A\left(\mathrm{~min}^{-1}\right)$ & 11700 & $2.9 \times 10^{14}$ & $1.69 \times 10^{13}$ \\
& $E_{\mathrm{a}}\left(\mathrm{kJ} \mathrm{mol}^{-1}\right)$ & 25.5 & 120 & 98 \\
Stopping & $A\left(\mathrm{~min}^{-1}\right)$ & 290 & $1.2 \times 10^{8}$ & $8.54 \times 10^{11}$ \\
& $E_{\mathrm{a}}\left(\mathrm{kJ} \mathrm{mol}^{-1}\right)$ & 26.7 & 80 & 106 \\
Alkaline & $A\left(\mathrm{~min}^{-1}\right)$ & 982 & 636 & $6.64 \times 10^{15}$ \\
hydrolysis & & & & \\
& $E_{\mathrm{a}}\left(\mathrm{kJ} \mathrm{mol}^{-1}\right)$ & 62.4 & 57 & 175 \\
\hline
\end{tabular}

Table 5: Estimated molalities of the initial REGs for the carbohydrates in Scots pine based on a combination of fitting the YL model to the YL data from kraft cooking and some a priori estimates of the initial DP.

\begin{tabular}{|c|c|c|c|}
\hline Carbohydrate & $\begin{array}{r}\text { Active REGs } \\
\left(\mu \mathrm{mol} \mathrm{g}^{-1}\right)\end{array}$ & $\begin{array}{r}\text { Stabilized } \\
\text { REGs }\left(\mu \mathrm{mol} \mathrm{g}^{-1}\right)\end{array}$ & DP \\
\hline Xylan & 56.8 & 0 & 133 \\
\hline GGM & 24.7 & 21.6 & 133 \\
\hline Cellulose & 3.1 & 0.6 & 1693 \\
\hline
\end{tabular}

(Jacobs and Dahlman 2001). The average of these three values was employed as an a priori estimate for both GGM and xylan. Table 5 shows the estimates for active and stabilized REGs. The parameter estimates the rate constants and the initial portion of REGs by fitting the YL model, which is inserted into the DecP model. Thus, the simulation of the time development is empowered for DP as well as the molalities of the active and stabilized REGs for the different carbohydrate chains as presented in Figure 3.

\section{Conclusions}

A coherent mathematical model describing simultaneously the YL and DecP for carbohydrates during alkaline treatment is conceivable. If both YL and DecP values are available, fitting the combined model provides a joint estimate of the rate constants and the initial REGs. The combined model applied on the YL data alone, together with some a priori knowledge of the initial average polymer chain length, provides a means for assessing the time development of the DecP as well as the active and stabilized REGs.

In the case of soda-AQ treatment of cotton linters, primary peeling dominates the rapid YL during the first few minutes and secondary peeling dominates the YL later on. Alkaline hydrolysis has an insignificant direct effect on the YL, but it is essential in triggering secondary peeling. The rate constants for peeling, stopping, and alkaline hydrolysis do not change as a function of the different pretreatments; the initial portions of active and stabilized REGs alone account for the differences in YL and DP. Both alkaline hydrolysis and peeling contribute substantially to the reduction of DP, especially in the beginning. A model of DP during alkaline treatment must take peeling into consideration. OA pretreatment increases the number of active REGs. Subsequent BH or AQS pretreatment accomplishes a strong increase in the stabilized REGs.

If Scots pine was submitted to kraft treatment, the temperature has a greater effect on xylan and cellulose DP than that of GGM. Of the three carbohydrates, xylan has the lowest and GGM has the highest initial ratio between stabilized and active REGs.

Acknowledgments: The authors acknowledge the Finnish Bioeconomy Cluster Oy (FIBIC), The Finnish Funding Agency for Technology and Innovation (Tekes), Andritz Oy, Danisco Sweeteners Oy, Metsä Fibre Oy, Stora Enso Oyj, UPM, and the International Doctoral Programme in Bioproducts Technology for financial support.

\section{References}

Calvini, P. (2012) The role of the Ekenstam equation on the kinetics of cellulose hydrolytic degradation. Cellulose 19:313-318.

Calvini, P. (2013) On the meaning of the Emsley, Ding \& Wang and Calvini equations applied to the degradation of cellulose. Cellulose 21:1127-1134.

De Groot, B., Van Dam, J.E.G., Van't Riet, K. (1995) Alkaline pulping of hemp woody core: kinetic modelling of lignin, xylan and cellulose extraction and degradation. Holzforschung 42:332-342.

Glaus, M.A., Van Loon, L.R. (2008) Degradation of cellulose under alkaline conditions: new insights from 12 years degradation study. Environ. Sci. Technol. 42:2906-2911.

Glaus, M.A., Van Loon, L.R., Achatz, S., Chodura, A., Fischer, K. (1999) Degradation of cellulosic materials under the alkaline conditions of a cementitious repository for low and intermediate level radioactive waste. Part I: identification of degradation products. Anal. Chim. Acta 398:111-122.

Ekenstam, A. (1936) The behavior of cellulose in mineral acid solution: kinetic study of the decomposition of cellulose in acid solutions. Ber. Dtsch. Chem. Ges. 69:553-559.

Haas, D.W., Hrutford, B.F., Sarkanen, K.V. (1967) Kinetics of alkaline hydrolysis of glycosidic bonds in cotton hydrocellulose. J. Appl. Polym. Sci. 11:113-116.

Jacobs, A., Dahlman, O. (2001) Characterization of the molar masses of hemicelluloses from wood and pulps employing size exclusion chromatography and matrix-assisted laser desorption 
ionization time-of-flight mass spectrometry. Biomacromolecules 2:894-905.

Jafari, V., Sixta, H., van Heiningen, A. (2014) Kinetics of oxygen delignification of high-kappa pulp in a continuous flow-through reactor. Ind. Eng. Chem. Res. 53:8385-8394.

Knill, C.J., Kennedy, J.F. (2003) Degradation of cellulose under alkaline conditions. Carbohydr. Polym. 51:281-300.

Lai, Y.-Z., Sarkanen, K.V. (1967) Kinetics of alkaline hydrolysis of glycosidic bonds in cotton cellulose. Cellul. Chem. Technol. 1:517-527.

Longue Jr., D., Ayoub, A., Vendetti, R.A., Jameel, H., Colodette, J.L., Chang, H.-M. (2013) Ethanol precipitation of hetero-polysaccharide material from hardwood by alkaline extraction prior to the kraft cooking process. Bioresources 8:5319-5332.

Mozdyniewicz, D.J., Nieminen, K., Sixta, H. (2013) Alkaline steeping of dissolving pulp. Part I: cellulose degradation kinetics. Cellulose 20:1437-1451.

Nieminen, K., Paananen, M., Sixta, H. (2014) Kinetic model for carbohydrate degradation and dissolution during kraft pulping. Ind. Eng. Chem. Res. 53:11292-11302.

Paananen, M., Tamminen, T., Nieminen, K., Sixta, H. (2010) Galactoglucomannan stabilization during the initial kraft cooking of Scots pine. Holzforschung 64:683-692.

Paananen, M., Liitiä, T., Sixta, H. (2013) Further insight into carbohydrate degradation and dissolution behavior during kraft cooking under elevated alkalinity without and in the presence of anthraquinone. Ind. Eng. Chem. Res. 52:12777-12784.

Pakkanen, H., Paloheimo T., Alen R. (2013) Characterization of dissolved material during the initial phase of softwood kraft pulping. Tappi J. 12:37-43.

Pavasars, I., Hagberg J., Borén H., Allard B. (2003) Alkaline degradation of cellulose: mechanisms and kinetics. J. Polym. Environ. 11:39-47.

Procter, A.R., Apelt, H.M. (1969) Reactions of wood components with hydrogen sulfide. III. Efficiency of hydrogen sulfide pretreatment compared to other methods for stabilizing cellulose to alkaline degradation. Tappi 52:1518-1522.

Rout, S.P., Radford J., Laws, A.P., Sweeney, F., Elmekawy, A., Gille, L.J., Humphreys, P.N. (2014) Biodegradation of the alkaline cellulose degradation products generated during radioactive waste disposal. PLoS ONE 9:e107433/1-e107433/10.
Shatalov, A.A., Pereira, H. (2005a) Kinetics of polysaccharide degradation during ethanol-alkali delignification of giant reed - part 1. Cellulose and xylan. Carbohydr. Polym. 59:435-442.

Shatalov, A.A., Pereira, H. (2005b) Kinetics of polysaccharide degradation during ethanol-alkali delignification of giant reed - part 2. Minor carbohydrates and uronic acids. Carbohydr. Polym. 61:304-313.

Sixta, H., Potthast, A., Krotschek, A.W. (2006) Chemical pulping processes. In: Handbook of Pulp. Ed. Sixta, H. Wiley-VCH Verlag GmbH, Weinheim. pp. 109, 197.

Sjöström, E. (1977) The behavior of wood polysaccharides during alkaline pulping processes. Tappi 60:151-157.

Sjöström, E. (1993) Wood Chemistry. Fundamentals and Applications. 2nd ed. Academic Press, San Diego. pp. 51-71.

Testova, L., Nieminen, K., Penttilä, P., Serimaa R., Potthast, A., Sixta H. (2014) Cellulose degradation in alkaline media upon acid pretreatment and stabilization. Carbohydr. Polym. 100:185-194.

Van Heiningen, A., Ji, Y. (2012) Southern pine oxygen delignified pulps produced in a Berty throughflow reactor: how to obtain the highest degree of delignification while maintaining pulp yield and quality. Tappi J. 11:6-18.

Van Heiningen, A., Gao, Y., Da Silva Perez, D. (2002) Reduction of alkaline pulp yield from the mass fraction and degree of polymerization of cellulose. In: 7th EWLP Conference, Turku, Finland. pp. 63-68.

Van Heiningen, A., Tunc, M.S., Gao, Y., Da Silva Perez D. (2004) Relationship between alkaline pulp yield and the mass fraction and degree of polymerization of cellulose in the pulp. J. Pulp Pap. Sci. 30:211-217.

Van Loon, L.R., Glaus M.A. (1997) Review of the kinetics of alkaline degradation of cellulose in view of its relevance for safety assessment of radioactive waste repositories. II. J. Environ. Polym. Degrad. 5:97-108.

Wei, W., Li, L., Chang, L., Wang, Z. (2013) Chemical and structural characterization of alkaline-extractable hemicelluloses from various eucalyptus species. J. Appl. Polym. Sci. 130: 2390-2398.

Young, R.A., Liss, L. (1978) A kinetic study of the alkaline endwise degradation of gluco- and galactomannans. Cellul. Chem. Technol. 12:399-411. 